\title{
A Perlustration of Various Image Segmentation Techniques
}

\author{
Sonam Mandiratta \\ Department of computer \\ Science Amity University, \\ Haryana
}

\author{
Pooja Batra Nagpal \\ Assistant Professor \\ Department of Computer \\ Science Engineering \& \\ Technology \\ Amity University, Haryana
}

\author{
Sarika Chaudhary \\ Assistant Professor \\ Department of Computer \\ Science \\ Engineering \& Technology \\ Amity University, Haryana
}

\begin{abstract}
Image segmentation plays the main role in image processing. Segmentation defines as a process of splitting an image into multiple parts or multiple regions in order to analyze them. The aim of segmentation is to modify an image in such a way so that it can easy to analyze as well as understand. To analyze the different type of an Image in image processing, image segmentation plays a key step in it. The purpose of segmentation is to find out the meaningful information from an image. Image segmentation is also used to differentiate the various objects that are occurring in an image. Several images Segmentation techniques have been developed due to its importance in image processing. These segmentation techniques are also useful to make an image smooth as well as easy to evaluate. There are many techniques in image segmentation process and these techniques are Edge based, Region based, Thresholding based, ANN based, Fuzzy based, clustering based and watershed based that are discussed in this paper.
\end{abstract}

\section{Keywords}

Segmentation, Gray Histogram, Gradient, Region Growing, watershed transform, Global and Local thresholding.

\section{INTRODUCTION}

Image Segmentation partitioned the whole image into multiple parts in order to find out the meaningful information. This process is work on the behalf of some features which are Grayscale image, threshold value, RGB image, etc. [1]. Due to the importance of image segmentation, it is used in many fields like removing the unwanted noise from an image, medical images, machine vision, satellite images, computer vision, military, biometrics, Image Retrieval, features extraction and objects recognition etc. Till now, there is no perfect technique for image segmentation which can be applied on all type of images. It is a very difficult task to find out the best suitable segmentation technique which can be applied to a particular type of image. Since it is not necessary if a particular technique applied to one image remains applicable to another image.[4] Therefore, image segmentation has been classified into three categories like classical, AI (Artificial Intelligence) and hybrid technique. Using filters to remove the unwanted noise of an image is the an important part of image segmentation. [4] For enhancing the image as well as improve the quality of image, denoising means removing the unwanted noise is done before in segmentation process. On the basis of color, images are splitted into two types which are intensity and RGB images [10].The segmentation techniques which are mostly used Edge based segmentation, Fuzzy theory based segmentation, Artificial Neural Network (ANN) based segmentation,
Threshold based image segmentation, Region based image segmentation, Clustering based segmentation and Watershed based segmentation. [2].

\section{IMAGE SEGMENTATION TECHNIQUES}

Image segmentation has deliberated as a vital part in image based applications. An application, such as image recognition, image compression, we cannot implement the full image directly for the cause that it is incompetent and unfeasible.

In these types of applications image segmentation is needed. Consequently, various image segmentation techniques were recommended to segment an image before recognition or compression process $[3,4]$. The assortment of the particular segmentation technique depends on the image type as well as environment of the problem. Selection of the segmentation techniques are based on the two factors that are discontinuity principle or similarity $[3,6]$.

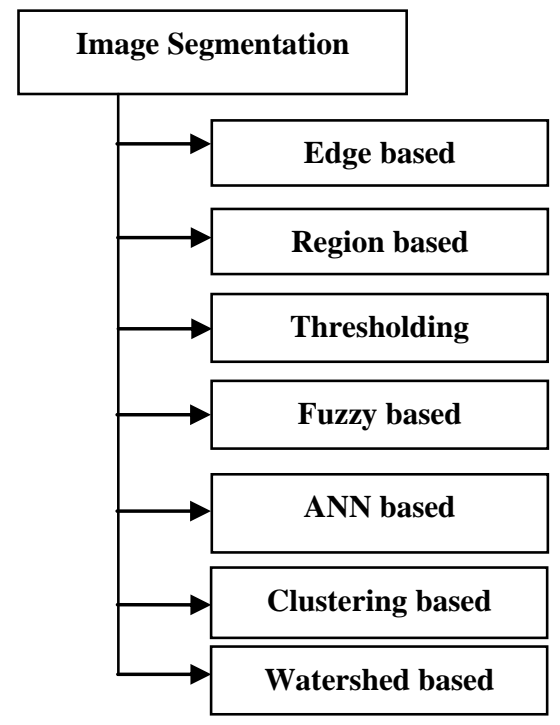

Fig 1: Flow diagram of various segmentation techniques.

\subsection{Edge based segmentation}

In image processing field, edge plays a vital role. Edge defines as a boundary of an object [7]. It is used to detect the object boundaries in image segmentation process. Edge based segmentation depends on the edges that are found in an image by using edge detecting operators like Roberts, prewitts, sobel, LOG, canny etc. There are two categories of edge detecting operators which are define as first order derivative and second order derivative. A group of connected pixels known as edge, that can be differentiated using intensity 
gradient estimation [3]. It divides the image by observing the changes in pixels on basis of their intensity value of an image in segmentation process. Edges are detected when there is suddenly change in the brightness of image. Gray Histogram and Gradient are two methods of edge detection that are commonly used in image segmentation [11].

\subsubsection{Gray histogram}

In this method, segmentation totally depends on choosing the accurate value of thresholding. The value of thresholding is either maximum or minimum and choosing the accurate value of threshold is very difficult because the gray histogram is alike for the images in which noise are existed if the value of thresholding is accurate then the quality of edge detection will also improve.[5.]

\subsubsection{Gradient}

Gradient is known as the first derivative of an image is. With the help of local maxima and local minima value gradient method is used to find out the edge of an image The most common edge operators are like Laplacian of Gaussian (LOG) operator, sobel operator, Laplace operator, and canny edge operator that are used in gradient methods. These operators are also known as first order derivative. In these operators, canny operator gives the best result as compared to other operators. When there is sudden change in intensity, then Gradient based method works well as compared to gray histogram and the result of segmentation is adaptive to the direction of gradient $[7,8]$.

\subsection{Region based segmentation}

This segmentation technique is also known as similarity based segmentation because segmentation is done based on the similarity of the given image. It divides an image into regions and that regions are compared on the basis of some predefined criteria. This approach uses the thresholding technique in order to separate the background from an image. This segmentation technique is very simple as compared to edge detection techniques. In this approach, each and every pixel is assigned to a particular region. The main purpose of region based segmentation is to produce such a region whose size is bigger and gives the result in some regions in the image $[3,8]$ The methods that are included in this technique are:-

\subsubsection{Region growing method}

Region growing method [7] is used for mining a particular image region which is connected that is based on some predefined criteria. This predefined criterion totally depends on intensity information.

The main purpose of this method is to select a seed point and grow regions from this seed pixel. Seed point is a pixel or we can say a point from where we begin. [6]

\subsubsection{Region splitting and merging}

This method works against to the region growing method.

Region splitting and merging is a top down approach because in this process the original image is divided into the regions that are not connected then merges it, while region growing is bottom-up approach because in which merging output is splitted according to the input. This approach works on whole image while region growing approach is not work on whole image. This approach is implemented with the quad tree data [2].

\subsubsection{Watershed transform}

The watershed transform is a mathematical tool that can be used in image segmentation. This tool is very powerful and it gives better result as compared to another segmentation techniques. Watershed transform has various benefits like it is simple, fast processing as well as gives the whole division of an image in separated regions even if the gap is minor. [9]

\subsection{Thresholding based segmentation}

It is a simple segmentation technique. In this technique, a definite value is taken that is called threshold used for measurement. It is used to segment the images which are having light objects on dark background. [11, 12] This technique is based on on the properties of an image. Thresholding based segmentation methods are:-

\subsubsection{Global thresholding}

This thresholding approach is used when the distribution of intensity will differ among foreground object and background. A particular threshold value is considered to separate the objects when the differences between these foreground and background objects are very different from each other $[1,6]$. The threshold value $(\mathrm{T})$ totally based on the pixel property and the intensity value of an image. The methods that are generally used in global thresholding are Otsu method, entropy based thresholding [6].

\subsubsection{Local thresholding}

In this thresholding method an image is separated into subpart and each sub-part is thresholded distinctly. This method works differ from previous method because this thresholding method used many threshold values and assigned to each subparts while in global thresholding only a single threshold value is used. Statistical thresholding, 2-D entropy-based thresholding and histogram transformation thresholding comes under this approach. [5, 7].

\subsection{Fuzzy based segmentation}

To analyze the any type of images as well as extract the useful data as per our requirement this techniques plays an important role. By default, unwanted noise occurred in an image, to remove it a function is used in this approach that is called Fuzzification function. For converting an intensity image into fuzzy image

Fuzzification function is used.[9]. For getting a better result, different morphological operators are collective with fuzzy method. There are many approaches that are included in this method are following:-

\subsubsection{Fuzzy Clustering Algorithm}

This fuzzy clustering approach is very ancient to segmentation. The generally used algorithms are Fuzzy kMeans and Fuzzy C-means (FCM) that are used to make the clusters [3]

\subsubsection{Fuzzy Rule-Based Approach}

To integrate the relationship among pixels, fuzzy rule-based approach is used. In this method a function is used which is having three types that are used to segmenting an image, that function is called membership function. Membership function's types are: Region pixel distribution, to measure the nearness of the region, and to find the $3 \mathrm{D}$ relationship between pixels [4].

2.4.3 Fuzzy connectedness using dynamic weights The customary segmentation outlines can't resolve the difficulties that are related to fuzzy medical images. For 
resolving these difficulties, they introduce a new algorithm that is known as Dynamic weight (DyW) algorithm which vigorously corrects the exact weights. This algorithm is also applicable in distingue images types [11].

\subsection{ANN based segmentation}

ANN stands for artificial neural network. It is also called as a neural network or neural net. Neural net is a synthetic representation of human brain that tries to pretend its learning procedure. For decision making process, neural net is also used. In recent centuries; ANN have been extensively used to resolve the difficulties that are still occur in medical image segmentation. It is depends on repetition of life, mainly the learning process of human brain, that forms a large number of equivalent nodes [2]. This equivalent node can implement the fundamental computing. To carrying the connections among nodes and connection weights, learning process should be done The benefit of ANN based segmentation technique is that, it is not based on a probability density distribution function (PDD). When the data move away from the original position it could not affect the segmentation result. [3]. To reduce the necessities of snooping in image segmentation process, neural net is used. It could also use to reduce the proficient interference requests. The purpose of choosing the connecting among the nodes and weights among the nodes, neural net was expert with ordered of training model [9]. This segmentation approach consist of two stages-

\subsubsection{Feature extraction}

This stage describes the ANN's data. It mined the features that are essential, this mined features are also helps in segmentation process.

\subsubsection{Neural network dependent image \\ Segmentation}

This stage performs the segmentation behalf on the essential features that are extracting from an image. Basically, It has three features:-

(i) Fast calculating and equivalent calculating's ability build it unable to work on real ground.

(ii) When the data move out from their normal position, better segmentation results are carried out.

(iii) Great toughness creates it exempt to noise [8, 18].

\subsection{Clustering based segmentation: The} concept of clustering is used in many areas like data mining, Image processing etc. Clustering is an unverified knowledge profession, at which the necessities to comprehend a limited set of classifications known as clusters to detect thelpixels. No training stages are cast-off in this unverified clustering [1]. A parallel condition is defined among the pixels and then these pixels are merged to construct the clusters. These Parallel conditions contain the image's elements which are dimension, RGB, surface etc. [17]. The cluster's quality behalf on two factors, which criteria is used and how this criterion is applied. The core determination of clustering algorithm is to return the good segmentation result. The commonly clustering methods are hard clustering, k-means clustering, fuzzy clustering, etc.

\subsubsection{Hard clustering}

Hard clustering method considers that an image's pixels belong to one cluster and boundaries are also among each cluster. K-mean algorithm is a hard clustering algorithm that is used mostly $[4,12]$. $\mathrm{K}$-mean clustering is a clustering method that collects the $\mathrm{n}$ as no. of pixels of an image into $\mathrm{k}$ number of clusters where no. of clusters(k) is less than no. of pixels(n) means $\mathrm{k}<\mathrm{n}$ and the value of $\mathrm{k}$ is positive. Primarily the centroids of the predefined clusters are set arbitrarily [6].Clusters is designed from the peak with nearly features that are pixel's gray level intensity and pixel intensity's distance [2]. K-means clustering algorithm is explained below:-

(i) Arbitrarily pick the K's value.

(ii) Arbitrarily select $\mathrm{K}$ pixels that are dissimilar intensity act as Centroids.

(iii) Centroids are ruling out by computing mean of pixel values in a region.

(iv) Relate the k's pixel to each Centroid and allocate the pixel to the Centroid that is very close to it and form the clusters. When all the pixels have been assigned, the basic clustering has been done.

(v) Again calculate the each cluster's mean as well as position of Centroids that in K Clusters.

(vi)Till all the Centroids are not stretched, continue the step (iv) and (v). [3]

\subsubsection{Fuzzy clustering}

When the boundaries among dissimilar objects of an image. Is not mentioned, in that situation Fuzzy clustering is used. It splits the input pixels into clusters on the origin of similarity measures that are space, connectivity, and intensity. [5] The benefits of this clustering are that, it is easily understandable because it uses fuzzy set. Possibility c-means(PCM) and Fuzzy c means(FCM) algorithm,GK(Gustafson-Kessel),GMD (Gaussian mixture decomposition), FCV (Fuzzy C varieties), AFC, FCS, FCSS, FCQS, FCRS all these algorithms are most generally used fuzzy clustering algorithms.[1] To preserve the many information FCM algorithm is considering as compared to other clustering algorithms. [2,6]

\subsection{Watershed based segmentation}

Watershed transformation is an apparatus that is used for image segmentation. It is established on the association process. One of the most apparatus used for image segmentation is watershed transform [20]. This apparatus can be used in image processing field, image segmentation that is a part of image processing. The benefits of watershed based segmentation is that it can be apply on any type of image like intensity, binary as well as texture image, high processing speed and it also produce the complete division of the image is compared to other image segmentation techniques It is a part of region-based segmentation technique. [21].To implements the watershed, three methods are defined:-

\subsubsection{Distance transforms approach}

This approach offers a range of the splitting the pixels in

an image. A function is used that is known as (bwdist) that is used to find out the distance among each pixel of an image that is set to either off or on [10].

\subsubsection{Gradient method}

This method used a function that is called as gradient magnitude function which is used to preprocess an intensity image earlier to use the watershed transform for segmenting an image. After applying this function to an image outputs comes in either high pixel values or low pixel values. The high pixel values of an image are occurring along object edges and low pixel values are everywhere except object edges. The 
procedure of this method to perform the segmentation is below: - firstly, find out the edges of an image that is being processed, and then apply watershed of the gradient. The weakness of this approach is over-segmentation [14]. To reduce this weakness another method is proposed that is marker controlled method. [21]

\subsubsection{Marker controlled method}

When the watershed transforms method implemented to the gradient image without non-stop, over-segmentation problem comes. To overcome this problem a method is presented that is known as marker. A marker is a linked constituent that suitable to an image. Markers are used to recover the limitation of gradient method. The two types of marker are internal markers and external markers. To find out the objects of an image, internal markers are used. While to find out the boundary of an image, external markers are used. [15, 20]

Table1: Comparison of various segmentation techniques.

\begin{tabular}{|c|c|c|}
\hline $\begin{array}{c}\text { Segmentation } \\
\text { Techniques }\end{array}$ & Pros. & Cons. \\
\hline $\begin{array}{c}\text { Edge based } \\
\text { Segmentation }\end{array}$ & $\begin{array}{l}\text { It works on those } \\
\text { images which } \\
\text { have best contrast } \\
\text { between objects } \\
\text { and background } \\
\text { of an image. }\end{array}$ & $\begin{array}{c}\text { It does not work on } \\
\text { those images which } \\
\text { have low contrast as } \\
\text { well as edges are not } \\
\text { defined in a proper } \\
\text { manner. }\end{array}$ \\
\hline $\begin{array}{l}\text { Region based } \\
\text { segmentation }\end{array}$ & $\begin{array}{l}\text { It works well on } \\
\text { noisy images as } \\
\text { compared to edge } \\
\text { based approach. }\end{array}$ & $\begin{array}{l}\text { It is more expensive } \\
\text { in calculating the } \\
\text { computational time } \\
\text { as well as memory. }\end{array}$ \\
\hline $\begin{array}{l}\text { Thresholding } \\
\text { based } \\
\text { segmentation }\end{array}$ & $\begin{array}{c}\text { It is simple } \\
\text { method and } \\
\text { mostly used for } \\
\text { segmentation. It is } \\
\text { also used in real- } \\
\text { time applications. }\end{array}$ & $\begin{array}{l}\text { Highly noise } \\
\text { sensitivity. } \\
\text { Choosing -an } \\
\text { accurate value of } \\
\text { threshold is a very } \\
\text { difficult task. }\end{array}$ \\
\hline $\begin{array}{c}\text { Fuzzy based } \\
\text { segmentation }\end{array}$ & $\begin{array}{c}\text { Fuzzy } \\
\text { membership } \\
\text { function can also } \\
\text { be used to display } \\
\text { the properties of } \\
\text { an image. }\end{array}$ & $\begin{array}{c}\text { To determine the } \\
\text { Fuzzy membership } \\
\text { function is a critical } \\
\text { part. }\end{array}$ \\
\hline $\begin{array}{l}\text { ANN based } \\
\text { segmentation }\end{array}$ & $\begin{array}{l}\text { Used to solve the } \\
\text { complex } \\
\text { problems. }\end{array}$ & $\begin{array}{l}\text { It requires apriori } \\
\text { learning parameter. } \\
\text { Training time is } \\
\text { lengthy. }\end{array}$ \\
\hline $\begin{array}{c}\text { Clustering based } \\
\text { segmentation }\end{array}$ & $\begin{array}{c}\text { K-means } \\
\text { clustering works } \\
\text { faster for small } \\
\text { values of k. } \\
\text { Remove the noisy } \\
\text { parts. }\end{array}$ & $\begin{array}{l}\text { Difficult to estimate } \\
\text { the fixed no. of } \\
\text { clusters. } \\
\text { It is expensive. }\end{array}$ \\
\hline $\begin{array}{c}\text { Watershed based } \\
\text { segmentation }\end{array}$ & $\begin{array}{l}\text { Its computation } \\
\text { speed is fast. }\end{array}$ & over segmentation \\
\hline
\end{tabular}

\section{CONCLUSION}

In this review paper various methods of image segmentation have been described, an outline of all related image segmentation approaches have been presented in this review paper. These image segmentation methods are discussed in this paper in recent technologies such as face identification, image identification, pattern recognition, image deblurring, disease detection etc. After the inspection of different methods of image segmentation, it is noticed that these methods may be used in many advanced fields such as for identification of regions images or object. Based on investigation, the conclusion is that image segmentation has a favorable future and a lot of work is necessary for evolving a best segmentation technique which has an ability to applied universally. For getting a better result of image segmentation we can also use hybrid approaches that means combining two approaches.

\section{REFERENCES}

[1] Sandhya, Archana, Poonam Rani "Image SegmentationMethods: A Review" International Journal of Advanced Research in Computer Science and Software EngineeringVolume 5, Issue 4, 2015.

[2] Priyanka Shivhare, Vinay Gupta "Review of Image Segmentation Techniques Including Pre \& Post Processing Operations "International Journal of Engineering and Advanced Technology (IJEAT) ISSN: 2249 - 8958, Volume-4 Issue-3, February 2015.

[3] M.S. Sonawane, C.A. Dhawale "A Brief Survey on Image Segmentation Methods "International Journal of Computer Applications (0975 - 8887) National conference on Digital Image and Signal Processing, DISP 2015 .

[4] Kamal Kant Verma, "A comparative study of image segmentation techniques in digital image processing" National Conference on "Emerging Trends in Electronics \& Communication". Special Issue, Vol. 1, No. 2, July 2015.

[5] Rohan Kandwal, Ashok Kumar, and Sanjay Bhargava "Review: Existing Image Segmentation Techniques" International Journal of Advanced Research in Computer Science and Software Engineering, Volume 4, Issue 4, April 2014.

[6] Ranjita Asati, H.R. Turkar, A.V. Anjikar, Chandu Vaizdya, Prashant Khobragade "A Survey On Spatial Based Image Segmentation Techniques" International Journal of Innovative Research in Computer and Communication Engineering Vol. 3, Issue 10, October 2015.

[7] M. Jogendra Kumar, Dr. GVS Raj Kumar and R. Vijay Kumar Reddy "Review on image segmentation techniques International Journal of Scientific Research Engineering \& Technology (IJSRET), ISSN 2278 0882Volume 3, Issue 6, September 2014.

[8] Amanpreet kaur, Navjot kaur "Image Segmentation Techniques" International Research Journal of Engineering and Technology (IRJET) Volume: 02 Issue: 02 | May-2015.

[9] S.S Sudha and K. K. Rahini, "Review of Image Segmentation Techniques: A Survey, "in Pros, International Journal of Advanced Research in Computer Science and Software Engineering, Volume 4, Issue 7, July 2014 
[10] Pinaki Pratim Acharjya, Soumya Mukherjee and Dibyendu Ghoshal," Digital Image Segmentation Using Median Filtering and Morphological Approach" Volume 4, Issue 1, January 2014.

[11] H.P. Narkhede, "Review of Image Segmentation Techniques", International Journal of Science and Modern Engineering (IJISME) ISSN: 2319-6386, Volume-1, Issue-8, July 2013.

[12] A.M. Khan, Ravi. S, "Image Segmentation Methods: A Comparative Study", International Journal of Soft Computing and Engineering (IJSCE) ISSN: 2231-2307, Volume-3, Issue-4, September 2013.

[13] Sujata Saini and Komal Arora (2014), "A Study Analysis on the Different Image Segmentation Techniques", International Journal of Information \& Computation Technology, Volume 4, Number 14 (2014).

[14] Gurpreet kaur, Sumeet kaushik: Effect of image gradient as initial step of watershed approach published in IJARCSSE Volume 3, Issue- 2, and February 2013.

[15] Er.Samina Tahir Rizvi, Er. Mandeep Singh Sandhu and Er. Shan E Fatima "Image Segmentation using Improved Watershed Algorithm" International Journal of Computer Science and Information Technologies, Vol. 5 (2), 2014.

[16] Rajeshwar Dass, Priyanka, Swapna Devi, "Image Segmentation Techniques", IJECT Vol. 3, Issue 1, Jan. March 2012.

[17] Acharya J, Gadhiya S and Raviya K (2013), "Segmentation Techniques for Image Analysis: A Review", International Journal of Computer Science and Management Research, Vol. 2, pp. 2278-2733.

[18] Amza C (2012), “A Review on Neural Network-Based Image Segmentation Techniques", Defort University, Mechanical and Manufacturing Eng., the Gateway Leicester, LE1 9BH, pp. 1-23, United Kingdom.

[19] Jay Acharya, Sohil Gadhiya and Kapil Raviya, "Segmentation Techniques for Image Analysis: A Review", International Journal of Computer Science and Management Research, Vol 2 Issue 1, January 2013, Pg. 1218-1221.

[20] Aman Kumar sharma1 \& Anju bala "marker based watershed transformation for image segmentation" vol. 3 , issue 4, Oct 2013, 187-192

[21] Amandeep Kaur, Aayushi "Image Segmentation Using Watershed Transform" Volume-4, Issue-1, March 2014.

[22] Dhruven Prajapati, Jenish Gandhi, Kruti J. Dangarwala, "A comparative study of various Image segmentation techniques", Volume 1 Issue5, 2014

[23] ]Swati Matta, "Review: Various Image Segmentation Techniques", (IJCSIT) International Journal of Computer Science and Information Technologies, Vol. 5 (6), 7536$7539,2014$.

[24] Segmentation Techniques for Image Analysis IJAERS/Vol. I/ Issue II/January-March, 2012.

[25] Ravi S and A M Khan, "Operators Used in Edge Detection: A Case Study", International Journal of Applied Engineering Research, ISSN 0973-4562 vol. 7 No 11, 2012.
[26] Punam Thakare, "A Study of Image Segmentation and Edge-Detection Techniques", International Journal on Computer Science and Engineering (IJCSE), Vol. 3 No. 2 Feb 2011

[27] Siti Noraini Sulaiman, Nor Ashidi Mat Isa, "Denoisingbased Clustering Algorithms for Segmentation of Low Level Salt-and-Pepper Noise-Corrupted Images", IEEE Transactions On Consumer Electronics, Vol. 56, No. 4, November 2010

[28] Punam Thakare, "A Study of Image Segmentation an Edge-Detection Techniques", International Journal on Computer Science and Engineering (IJCSE), Vol. 3 No. 2 Feb 2011

[29] V. K. Dehariya, S. K. Shrivastava, R. C. Jain, "Clustering of Image Data Set Using K- Means and Fuzzy K-Means Algorithms", International conference on CICN, pp. 386- 391, 2010.

[30] K. K. Singh, A. Singh, “A Study of Image Segmentation Algorithms for Different Types of Images", International Journal of Computer Science Issues, Vol. 7, Issue 5, 2010 .

[31] S.Thilagamani, N.Shanthi, "A Novel Recursive Clustering Algorithm for Image Over segmentation", European Journal of Scientific Research, Vol.52, No.3, pp.430-436, 2011

[32] Mariela Azul Gonzalez, Gustavo Javier Meschino, Virginia Laura Ballarin, "Solving the Over segmentation problem in applications of Watershed Transform", journal of Biomedical Graphics and Computing, Vol. 3, No. 3, pp.29-40, 2013.

[33] Pinaki Pratim Acharjya, Dibyendu Ghoshal, "A Modified Watershed Segmentation Algorithm using Distances Transform for Image Segmentation", International Journal of Computer Applications, Volume 52- No.12, pp. 47-50, August 2012.

[34] S.S. Al-amri, N.V. Kalyankar and Khamitkar S.D, "Image Segmentation by Using Threshold Techniques", journal of computing, volume 2, issue 5, may 2010.

[35] Ms R.Saranya Pon Selvi et al Int.Journal of Engineering Research and applications ISSN: 2248-9622, Vol.4, Issue 3(version 1), March 2014, pp.429-434.

[36] S. Lakshmi and D. V. Sankaranarayanan, "A study of edge detection techniques for Segmentation computing approaches," IJCA Special Issue on Computer Aided Soft Computing Techniques for Imaging and Biomedical Applications" CASCT, 2010.

[37] H. G. Kaganami, Z. Beij, "Region Based Detection versus Edge Detection", IEEE Transactions on Intelligent information hiding and multimedia signal processing, pp. 1217- 1221, 2009.

[38] S.K Somasundaram, P.Alli," A Review on Recent Research and Implementation Methodologies on Medical Image Segmentation”, Journal of Computer Science 8(1): 170-174, 2012.

[39] W. X. Kang, Q. Q. Yang, R. R. Liang, "The Comparative Research on Image Segmentation Algorithms", IEEE Conference on ETCS, pp. 703-707, 2009. 
International Journal of Computer Applications (0975 - 8887)

Volume 139 - No.12, April 2016

[40] H. Zhang, J. E. Fritts, S. A. Goldman, and "Image Segmentation "Evaluation: A Survey of Unsupervised methods", computer vision and image understanding, pp.260-280, 2008.

[41] K. Parvati, B. S. Prakasa Rao and M. Mariya Das, "Image Segmentation Using Gray-Scale Morphology and
Marker-Controlled Watershed Transformation", Discrete Dynamics in Nature and Society, vol. 2008, pp. 1-8, 2008.

[42] Rafael C. Gonzalez, Richard E. Woods, Steven L.Eddins, "Digital Image Processing Using MATLAB," Second Edition, Gatesmark Publishing, 2009. 